\title{
A top-down scenario for the formation of massive Tidal Dwarf Galaxies
}

\author{
P.-A. Duc ${ }^{1,2}$, F. Bournaud ${ }^{3,1,4}$, and F. Masset ${ }^{1}$ \\ ${ }^{1}$ CEA/DSM/DAPNIA, Service d'Astrophysique, Saclay, 91191 Gif-sur-Yvette Cedex, France \\ e-mail: paduc@cea.fr \\ 2 CNRS, FRE 2591 \\ 3 Observatoire de Paris, LERMA, 61 Av. de l'Observatoire, 75014 Paris, France \\ 4 École Normale Supérieure, 45 rue d’Ulm, 75005 Paris, France
}

Accepted 4 June 2004 / Accepted 9 August 2004

\begin{abstract}
Among those objects formed out of collisional debris during galaxy mergers, the prominent gaseous accumulations observed near the tip of some long tidal tails are the most likely to survive long enough to form genuine recycled galaxies. Using simple numerical models, Bournaud et al. (2003) claimed that tidal objects as massive as $10^{9} M_{\odot}$ could only form, in these simulations, within extended dark matter (DM) haloes. We present here a new set of simulations of galaxy collisions to further investigate the structure of tidal tails. First of all, we checked that massive objects are still produced in full $N$-body codes that include feedback and a large number of particles. Using a simpler $N$-body code with rigid haloes, we noticed that dissipation and self-gravity in the tails, although important, are not the key factors. Exploiting toy models, we found that, for truncated DM haloes, material is stretched along the tail, while, within extended haloes, the tidal field can efficiently carry away from the disk a large fraction of the gas, while maintaining its surface density to a high value. This creates a density enhancement near the tip of the tail. Only later-on, self-gravity takes over; the gas clouds collapse and start forming stars. Thus, such objects were fundamentally formed following a kinematical process, according to a top-down scenario, contrary to the less massive Super Star Clusters that are also present around mergers. This conclusion leads us to introduce a restrictive definition for Tidal Dwarf Galaxies (TDGs) and their progenitors, considering only the most massive ones, initially mostly made of gas, that were able to pile up in the tidal tails. More simulations will be necessary to precisely determine the fate of these proto-TDGs and estimate their number.
\end{abstract}

Key words. galaxies: formation - galaxies: interactions - galaxies: dwarf - galaxies: halos - cosmology: dark matter

\section{Introduction}

Numerous papers have reported the formation of presumably bound stellar structures around interacting systems. Their blue colors and high metallicity indicate that they were formed rather recently out of material processed in the disk of their parent galaxies. The census of young objects in mergers is still on going and their taxonomy not yet completed.

- Super Star Clusters (SSCs), with typical masses of $10^{5} M_{\odot}$, appear on optical images as compact stellar condensations. They are either located in the central regions of mergers (e.g., Holtzman et al. 1992; Whitmore et al. 1999; Zepf et al. 1999), in tidal bridges or along extended tidal tails (e.g., Gallagher et al. 2001; Knierman et al. 2003; Saviane et al. 2004) and may be dust enshrouded (Gilbert et al. 2000). Young Massive Clusters (YMCs) were identified in high-resolution HST images (e.g., de Grijs et al. 2003; Tran et al. 2003). Their blue luminosity, inferred mass and size are such that some of them may evolve into Globular Clusters with typical masses of $10^{6} M_{\odot}$ (e.g., Schweizer et al. 1996). Some $10^{7} M_{\odot}$
HI fragments discovered around mergers could be their gaseous progenitors (English et al. 2003).

- Giant HII Complexes (GHCs), i.e. star-forming regions with luminosities exceeding the Giant HII Regions found in isolated spiral disks, were discovered in long optical tidal tails (e.g., Weilbacher et al. 2003; López-Sánchez et al. 2004). Further away, Intergalactic Emission Line Regions, characterized by a very low underlying old stellar content, and sometimes by their compact aspect (the so-called EL-Dots) were detected in several clusters of galaxies. Their optical spectra are typical of star-forming HII regions and their rather high oxygen abundances indicate that they are formed of pre-enriched gas probably stripped from parent colliding galaxies (e.g., Gerhard et al. 2002; Cortese et al. 2004; Ryan-Weber et al. 2004; Mendes de Oliveira et al. 2004).

- Finally, the formation in tidal debris of Tidal Dwarf Galaxies (TDGs), i.e. objects with apparent masses and sizes of dwarf galaxies, has been reported in several studies of interacting systems (e.g., Duc \& Mirabel 1999; Hibbard \& Barnes 2004, and references thereein). They contain large quantities of gas in 
atomic, molecular and ionized form and have luminous masses of typically $10^{9} M_{\odot}$ (e.g., Braine et al. 2001). Because they are most often observed at or near the tip of long optical tidal tails, the very existence of such massive objects has been challenged. Indeed an apparent accumulation of tidal material could be artificial. In 3D space, tidal tails are curved. Seen edge-on, they appear as linear structures and may show at their tip fake mass concentrations caused by material projected along the line of sight (Hibbard \& Barnes 2004; Mihos 2004). Kinematical studies of tidal tails help in identifying such projection effects (Bournaud et al. 2004). The spatial and velocity coincidence of the different phases of the tidal gas towards a TDG candidate adds circumstantial evidence as to its reality (Braine et al. 2001).

These objects around mergers all contain stars with an age, derived from their colors or spectroscopic properties, which is comparable to or smaller than the dynamical age of the collision between the parent galaxies. They were hence most probably formed during the merging process. This does not mean however that a single mechanism is at their origin. Indeed, the heterogeneity of their properties (gaseous content, stellar population, location, and above all their luminosity/mass) rather suggests that several physical processes take place during the collision. The initial conditions of the encounter may also matter to account, for instance, for the formation of the most massive tidal objects, exceeding $10^{9} M_{\odot}$, i.e. those often referred as Tidal Dwarf Galaxies.

Numerical simulations of collisions are particularly helpful to identify the mechanism responsible for the formation of young objects in tidal debris. Soon after the study by Mirabel et al. (1992) of a Tidal Dwarf Galaxy candidate in the Antennae system, Barnes \& Hernquist (1992) and Elmegreen et al. (1993) published numerical models exhibiting bound condensations along tidal tails. While in the Barnes \& Hernquist (1992) simulations, the condensations formed from gravitational instabilities in the stellar component, those of Elmegreen et al. (1993) were produced directly from gas clouds, the velocity dispersion of which had increased due to the collision. Further simulations, made with a higher resolution and/or more realistic initial conditions, especially regarding the respective distribution of the gaseous and stellar components, could produce along the tails objects likely to be the progenitors of SSCs or globular clusters (e.g., Barnes \& Hernquist 1996; Kroupa 1998; Bekki et al. 2002). Their masses of typically $10^{8} M_{\odot}$ are however one order of magnitude lower than that measured in the Tidal Dwarf Galaxies located at the tip of tidal tails.

In a few older simulations, however, the formation of massive accumulations of tidal material were noted (see Fig. 3 in Barnes 1992 or Fig. 3b in Elmegreen et al. 1993) but were not much commented as they were quickly disrupted or went out of the volume studied in the model. Elmegreen et al. (1993) actually concluded that massive galaxy interactions could lead to the ejection of the most outer HI disk, forming an "extended $10^{9} M_{\odot}$ pool at the end of the tidal tail". Recently, Bournaud et al. (2003) (hereafter BDM03) using a new set of simulations involving simple co-planar collisions showed that such large end-of-tail blobs could condense and become gravitationally bound. Varying the initial size of the dark matter (DM) haloes in the parent galaxies, they found that objects as massive as $10^{9} M_{\odot}$, i.e. similar to TDGs, could only be produced when the haloes were extended enough - at least ten times the optical radius. Most often, dark matter haloes are truncated in numerical simulations although cosmological models predict their large size. This trick saves a great number of DM particles and is justified as long as the central regions of colliding galaxies are studied. BDM03 actually found that the extent of the DM halo influences the internal structure of the tidal tails, i.e. the distribution of matter along them, while previous works had more focussed on the length and curvature of these tails (e.g., Mihos et al. 1998; Springel \& White 1999; Dubinski et al. 1999).

The simulations presented by BDM03 had a number of limits. The DM and stellar components were computed with a 3D FFT code at a resolution of only $5 \mathrm{kpc}$ while the gas was simulated in 2D (i.e. in a co-planar encounter) with a resolution of $150 \mathrm{pc}$ high enough to resolve the gaseous tidal tails. Thus, the possible role of stellar sub-structures (which could not form in the low-resolution code) in building TDGs was ignored. Beside, the gas/DM halo interaction was not taken into account. In this paper, we check the results of BDM03 in full 3D $N$-body simulations that have, beside, a much higher resolution in both the DM, stellar and gas components (Sect. 2). Having confirmed with this new code the early results of BDM03 and assessed the fundamental role of kinematical processes in structuring tidal tails, we use simpler simulations and qualitative arguments to clarify the effect of tidal forces within extended DM haloes (Sect. 3). Finally, in Sect. 4, we revisit the definition of Tidal Dwarf Galaxies, arguing that, being formed according to a top-down scenario, they have a different physical origin than the other tidal objects.

This paper only discusses the physics of the formation of massive TDGs. It does not address their abundance nor their evolution and survival time. Answering these issues of cosmological importance requires to carry out a large number of simulations with a comprehensive set of initial conditions. This work is in progress and will be presented elsewhere.

\section{Formation of TDGs in full 3D $\mathbf{N}$-body simulations}

We have carried out a series of full 3D $N$-body simulations of the merger of two spiral galaxies with mass ratios between 1:1 and 1.7:1 and different orbital parameters. Their resolution, which was much higher than in BDM03, allowed to precisely study the internal structure of the tidal tails.

\subsection{Code description}

\subsubsection{Physical model}

We have used the $N$-body FFT code of Bournaud \& Combes (2003). In the main simulation presented in detail hereafter, the number of particles for the most massive galaxy was $2 \times 10^{6}$ for dark matter, $10^{6}$ for stars, and $10^{6}$ for gas. For the other galaxy, the numbers were scaled to its mass. The gravitational potential was computed on a Cartesian grid of size $1024^{3}$, with a softening length of $390 \mathrm{pc}$. We have also run three other 
simulations with a four times smaller number of particles meshed on a $512^{3}$ grid, and a softening length of $780 \mathrm{pc}$.

The dissipative nature of the ISM has been modeled through the sticky-particles code of Bournaud \& Combes (2002), with elasticity parameters $\beta_{\mathrm{t}}=\beta_{\mathrm{r}}=0.8$ for cloudcloud collisions. Star formation was described by a generalized Schmidt law (Schmidt 1959), and we employed a timedependent stellar mass-loss model inspired of that of Jungwiert et al. (2001) and described in Bournaud \& Combes (2002). We then computed the star formation and mass-loss rates in each cell of the Cartesian grid, according to the values of these two rates, converting a fraction of gaseous particles into stellar ones and vice versa.

\subsubsection{Mass distribution of galaxies}

Each galaxy was made-up of a spherical dark halo, a stellar and gaseous disk, and a spherical bulge. For the most massive galaxy, with a visible mass of $2 \times 10^{11} M_{\odot}$, the stellar disk was a Toomre disk with a radius of $15 \mathrm{kpc}$, a radial scale-length of $5 \mathrm{kpc}$ and the same vertical distribution as in Bournaud \& Combes (2002). The gaseous disk is a Toomre disk of truncation radius $37.5 \mathrm{kpc}$ and a radial scale-length of $15 \mathrm{kpc}$. Such a gaseous disk which is 2.5 times more extended than the stellar one is representative of most spiral galaxies (e.g., Roberts \& Haynes 1994). The bulge was modeled by a Plummer sphere of scale-length $1 \mathrm{kpc}$ and truncation radius $3 \mathrm{kpc}$. The dark halo was a softened isothermal sphere truncated at radius $150 \mathrm{kpc}$ (10 times the stellar disk radius). Its density profile is given by:

$\rho(r)=\frac{\sigma^{2}}{2 \pi G\left(r^{2}+r_{\mathrm{c}}^{2}\right)}$.

We used a core radius $r_{\mathrm{c}}=4.5 \mathrm{kpc}$. For the other galaxy, the radial distribution of matter was scaled by the square root of its stellar mass. In all simulations, we chose a bulge to disk mass ratio of 0.3 , and a dark-to-visible mass ratio inside the stellar radius of 0.6 . The mass fraction of gas inside the disk has been varied (see Table 1).

\subsubsection{Galactic encounter and orbital parameters}

Table 1 lists the values of the orbital parameters used in our simulations. The ratio $M$ of the stellar masses between the two galaxies roughly corresponds to the ratio of the dark halo masses and hence of the total masses. We chose orbital parameters that favor the formation of a long tidal tail for at least one of the two galaxies; in particular we have only considered prograde orbits.

\subsection{Results}

Figure 1 presents the evolution of the gas distribution for simulation A. The stellar, gaseous and dark matter components are shown at key moments of the merging sequence in Figs. 2 and 3 .

In run $\mathrm{A}$, two major tidal tails of $\simeq 100 \mathrm{kpc}$ developed soon after the periapse (at $t=410 \mathrm{Myr}$ ). At $t=470 \mathrm{Myr}$, massive
Table 1. Run parameters.

\begin{tabular}{cccccccccc}
\hline \hline Run & $N^{a}$ & $M^{b}$ & $m_{\mathrm{g}}{ }^{c}$ & ${m_{\mathrm{g}}}{ }^{\mathrm{d}}$ & $V^{e}$ & $b^{f}$ & $i_{1}{ }^{g}$ & $i_{2}{ }^{h}$ & $\omega^{i}$ \\
\hline $\mathrm{A}$ & $1024^{3}$ & 1.2 & $8 \%$ & $10 \%$ & 150 & 90 & 15 & 20 & 27 \\
$\mathrm{~B}$ & $512^{3}$ & 1.7 & $5 \%$ & $12 \%$ & 150 & 50 & 45 & 20 & 40 \\
$\mathrm{C}$ & $512^{3}$ & 1.0 & $8 \%$ & $8 \%$ & 170 & 75 & 15 & 15 & 30 \\
$\mathrm{D}$ & $512^{3}$ & 1.3 & $9 \%$ & $9 \%$ & 120 & 120 & 25 & 25 & 35 \\
\hline
\end{tabular}

a Spatial resolution: $N$ is the number of cells on the Cartesian grid. See text for the associated number of particles and softening length.

${ }^{b}$ Stellar mass ratio.

${ }^{c}$ Gas mass fraction in the most massive galaxy; $m_{\mathrm{g}}$ is the gas to disk+bulge mass ratio.

${ }^{d}$ Gas mass fraction in the less massive galaxy.

${ }^{e}$ Relative velocity of the two galaxies computed at an infinite distance in $\mathrm{km} \mathrm{s}^{-1}$.

${ }^{f}$ Impact parameter in kpc.

${ }^{g}$ Inclination of the most massive galactic disk with respect to the orbital plane.

${ }^{h}$ Inclination of the less massive galactic disk with respect to the orbital plane.

${ }^{i}$ Angle between the two galactic disks.

gas clouds have piled up at the tip of each tidal tail: they are the progenitors of the TDGs. We find that $70 \%$ of the gas along the tails is located in the 20 last $\mathrm{kpc}$, while the tails are about $100 \mathrm{kpc}$ long $^{1}$. The total masses of the two TDG progenitors at $t=470 \mathrm{Myr}$, computed in spheres of diameter $20 \mathrm{kpc}$ centered on the TDG luminosity peak, are resp. $2.9 \times 10^{9} M_{\odot}$ and $2.3 \times$ $10^{9} M_{\odot}$ (see Table 2). At this stage, most of the mass is in the gas; the stars contribute to $25 \%$ of the mass. The majority of the stars were actually formed in situ in the tail. The old stellar population which was tidally ejected along with the gas contributes to only resp. $5 \%$ and $12 \%$ of the total mass.

At $t=500 \mathrm{Myr}$, the stellar disks have already merged. The two major condensations at the tip of the tails have become more compact due to the action of self-gravity. Smaller substructures developed along the two major tails as well as in the two smaller counter-tails. They do not survive later on either because they are tidally disrupted or because they simply fall back. At $t=840 \mathrm{Myr}$ (430 Myr after the periapse), the two TDGs, which now appear isolated, orbit around the merger remnant at a radius of about $100 \mathrm{kpc}$. Their respective masses are now $2.2 \times 10^{9} M_{\odot}$ and $1.8 \times 10^{9} \mathrm{M}_{\odot}{ }^{2}$. At the end of the simulations, the TDGs have converted a large fraction of their gas into young stars (see Fig. 3). The mass of old stars generally represents 5 to $15 \%$ of the mass of the TDG (see Table 2),

\footnotetext{
1 We computed the gas mass in a tail as the mass at radii larger than $30 \mathrm{kpc}$ from the galaxy center. We chose to consider for the mass of the TDG progenitor all the matter included within a fixed aperture, rather than using a friend-to-friend algorithm that would better delineate the bound tidal object. Indeed this is how the luminosity and mass are usually measured in real observations. Thus, the real mass may have slightly been overestimated, as in the reality. The initial limit of $20 \mathrm{kpc}$ was determined varying the aperture radius, which is also the method used for aperture photometry.

${ }^{2}$ One fourth on the mass of the condensations of gas measured at $t=470 \mathrm{Myr}$ thus turned out not to belong to the gravitationally linked object.
} 

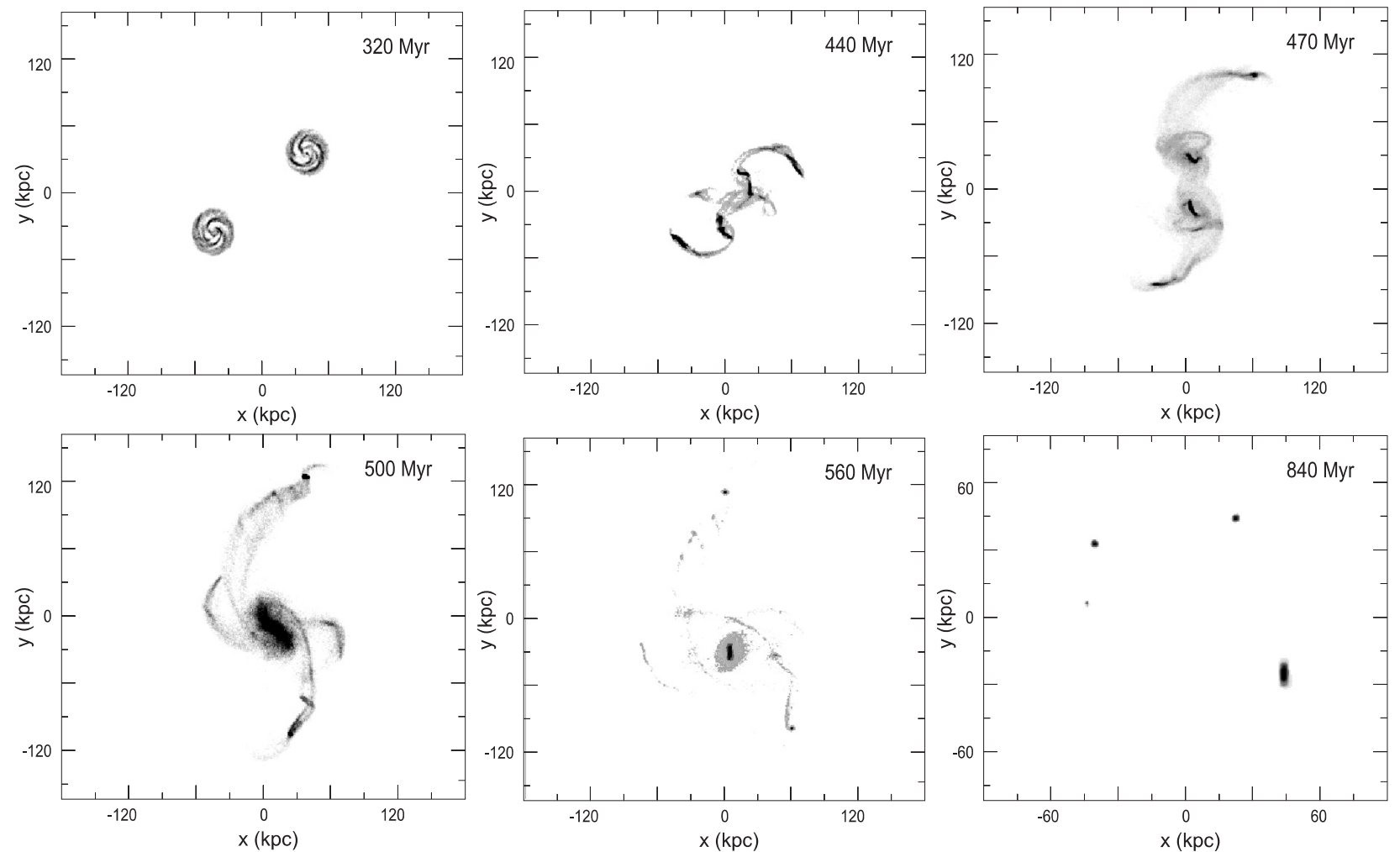

Fig. 1. Evolution of the gas distribution for the full $N$-body simulations of run A. The system is seen face-on. The gas is displayed with a logarithm intensity scale.

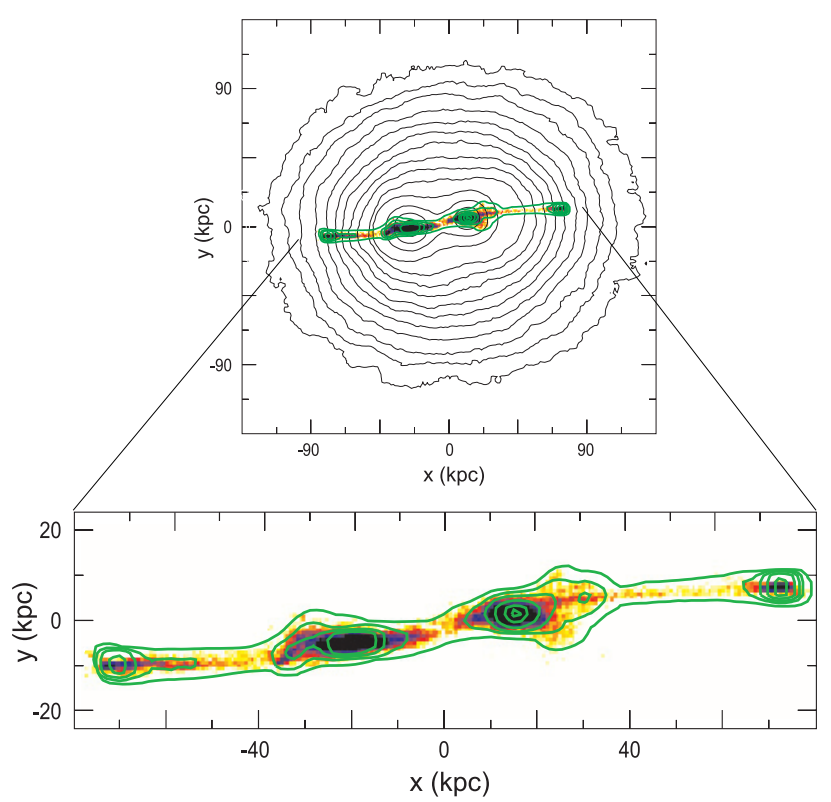

Fig. 2. Bottom: contours of the gaseous component superimposed on a grayscale representation of the stellar component at $t=470 \mathrm{Myr}$ (simulation A). Top: zoom-out with the contours of the dark matter haloes superimposed. The system is seen edge-on.

but this quantity may vary with the extent of the gaseous disk compared to the stellar one, which has not been changed here.

As many real interacting systems for which detailed studies of TDGs exist are observed close to edge-on, we present in Fig. 2 an edge-on view at a time when the TDGs have already

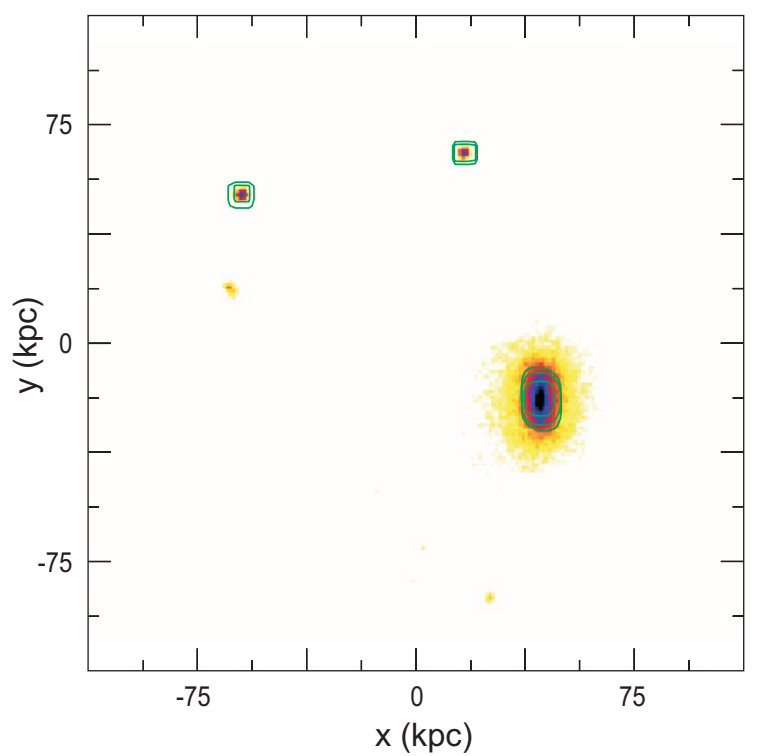

Fig. 3. Contours of the gaseous component superimposed on a grayscale representation of the stellar component at $t=850 \mathrm{Myr}$ (run A). The system is seen face-on.

formed. With this line of sight, the tidal tails appear as linear structures and the TDGs as prominent overdensities at their apparent tip. This is close to what is observed in systems like NGC 4676 (Hibbard \& van Gorkom 1996) or Arp 105 (Duc et al. 1997). The simulated systems seem however to have more narrow tidal features, especially in the stellar component. 

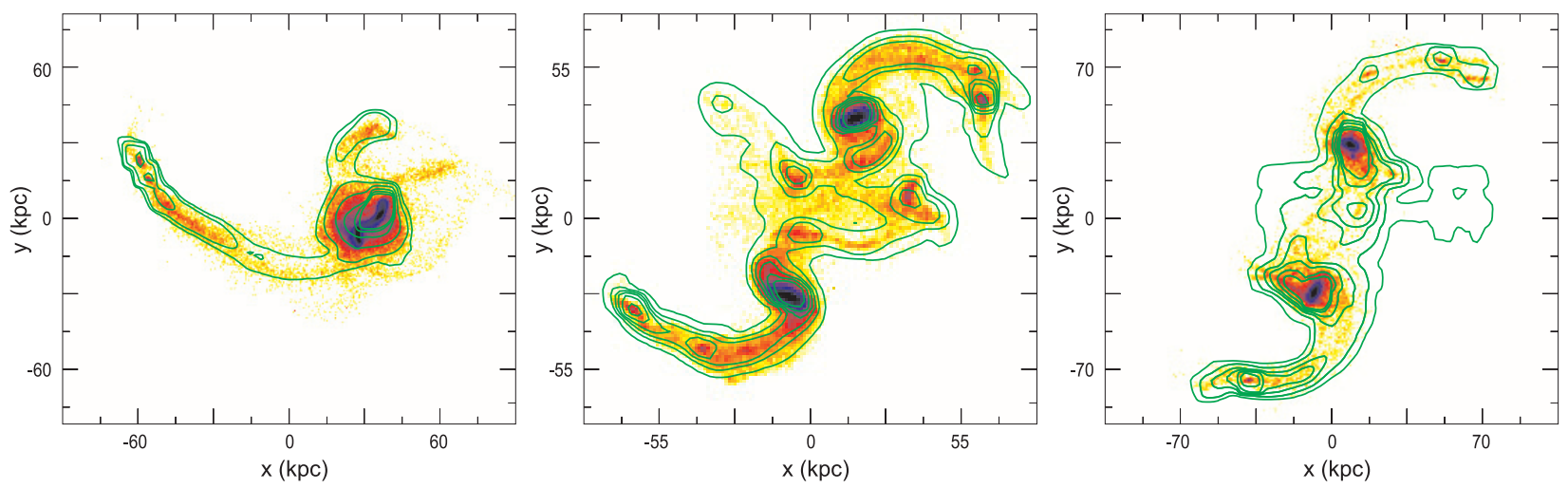

Fig. 4. Results of the full $N$-body simulations of runs B (left), C (middle) and D (right) 50 to 100 Myrs after the periapse (see Table 2). Gas contours are superimposed on the stellar component. All systems are seen face-on.

The fact that the mass is dominated by the gaseous component is also quite realistic. The HI mass of typical TDG candidates is indeed a factor of 2-5 higher than the stellar mass estimated from their optical luminosity (Braine et al. 2001).

Figure 4 presents snapshots 50 to $100 \mathrm{Myr}$ after the periapse for runs $\mathrm{B}, \mathrm{C}$ and $\mathrm{D}$ which were all carried out with the extended dark matter haloes. Despite their slightly different initial conditions, orbital parameters and a spatial resolution which is twice lower, they all show at the tip of the tails prominent gaseous condensations with masses well above $1.0 \times$ $10^{9} M_{\odot}$. Table 2 lists their properties. In run B, the longest tail gives birth to a gravitationally bound TDG, while the shortest tail exhibits a smooth accumulation of matter at its tip which is not gravitationally bound and only transient. In run $\mathrm{C}$, one of the major tidal accumulations fragmented into two pieces; the other one remains in the form of a single object. The two countertails (near $y=0$ on Fig. 4) also show accumulations of matter in their outer parts, but these ones do not survive as they fall back quickly onto the parent galaxies. Run D, as well as run $\mathrm{C}$, shows on top of the prominent TDG progenitor a few small stellar condensations, distributed along the tail, with masses of the order of $10^{8} M_{\odot}$ for the most massive ones; these gravitational clumps have similar masses as those produced in the simulations by Barnes \& Hernquist (1992) and Elmegreen et al. (1993). Like in Elmegreen et al. (1993), they were originally formed in gaseous condensations and generally less than $20-25 \%$ of their mass correspond to old stars expelled from the parent spiral disk. A few dynamical times later, they mostly consist of young stars formed in situ from the gas clumps. Hence they may be the progenitors of the SSCs observed in real interacting systems. However, a detailed comparison of the simulated and real SSC is beyond the scope of this paper.

We carried out a few other simulations, truncating this time the dark matter haloes. Sub-structures form along the tidal tails, but no massive TDG progenitor is produced. Because these simulations are totally similar to those carried out by the other groups (e.g., Barnes \& Hernquist 1992), they are not presented here.

Therefore, including the dynamical friction in full $\mathrm{N}$-body simulations and increasing their resolution did not change the
Table 2. TDGs progenitors.

\begin{tabular}{lccccc}
\hline \hline Run & Location & $\begin{array}{c}T^{a} \\
(\mathrm{Myr})\end{array}$ & $\begin{array}{c}M_{\mathrm{tot}}{ }^{b} \\
\left(M_{\odot}\right)\end{array}$ & $\begin{array}{c}M_{*}^{\text {old }} c \\
(\%)\end{array}$ & $\begin{array}{c}M_{*}^{\text {young } d} \\
(\%)\end{array}$ \\
\hline A & $x>0$ & 60 & $2.9 \times 10^{9}$ & $5 \%$ & $19 \%$ \\
& $x<0$ & & $2.3 \times 10^{9}$ & $12 \%$ & $13 \%$ \\
& $x>0$ & 430 & $2.2 \times 10^{9}$ & $5 \%$ & $44 \%$ \\
& $x<0$ & & $1.8 \times 10^{9}$ & $14 \%$ & $31 \%$ \\
\hline B & $x<0$ & 100 & $2.3 \times 10^{9}$ & $7 \%$ & $19 \%$ \\
\hline $\mathrm{C}$ & $x>0$ & 90 & $2.5 \times 10^{9} e$ & $11 \%$ & $16 \%$ \\
& $x<0$ & & $1.8 \times 10^{9}$ & $11 \%$ & $16 \%$ \\
\hline $\mathrm{D}$ & $y>0$ & 55 & $1.1 \times 10^{9}$ & $3 \%$ & $14 \%$ \\
& $y<0$ & & $2.4 \times 10^{9}$ & $8 \%$ & $19 \%$ \\
\hline
\end{tabular}

${ }^{a}$ Time after the periapse.

$b$ Total mass.

${ }^{c}$ Mass of the old stellar population from the parent's disk.

${ }^{d}$ Mass of the young stellar population born in situ. Note that this value is approximate as the measurement of the SFR is affected by the limited numerical resolution.

${ }^{e}$ In two fragments.

basic results regarding the formation of TDGs initially presented in BDM03: massive gaseous clouds are still formed near the end of the tidal tail in this, otherwise, much more realistic model.

\section{Mechanism for the formation of TDGs and role of the dark haloes}

We have found that dark haloes should be very extended to enable the formation of massive TDGs. Yet, since a more extended halo contains more mass, one may wonder whether the total DM mass is more important for the formation of the TDG precursors than its actual extent. We checked that in BDMD03 with simulations in which the truncated DM halo had a mass similar to the extended one. We were then unable to produce any massive accumulation in the tidal tails. Thus, 
the DM halo extent - and hence the shape of the corresponding potential well - appears as the critical parameter, rather than the mass of the DM halo. Dubinski et al. (1996) and Mihos et al. (1998) actually claimed that massive, dense, haloes are less able to form long-lived tidal tails and hence proto-TDGs. Mihos et al. (1998) noted however that it is actually "the shape and gradient of the galactic potential", rather than the mass of the DM halo, that determine the formation and evolution of tidal tails.

In order to decipher the various mechanisms relevant for the formation of TDGs and weight their relative importance, we carried out a new set of restricted $N$-body simulations. The full $N$-body simulations described in Sect. 2 ensured that, using simpler models that can be more easily analyzed, one would not disregard the main physical process. In this whole section, we denote as truncated a DM halo truncated at three times the stellar disk radius, and extended a DM halo extended up to ten times the stellar disk radius.

\subsection{A kinematical origin}

We first analyze the role of dissipation and self-gravity using a hybrid code: $N$-body for the gas, and 3-body for the other components. In 3-body codes, massless particles evolve in the rigid potential of two galaxies (Toomre \& Toomre 1972). Each galaxy potential was represented by a rigid rotation inside the central kpc, by a flat rotation curve for radii from 1 to $150 \mathrm{kpc}$, and a Keplerian potential at radii larger than $150 \mathrm{kpc}$. Such a profile corresponds to the case where the dark matter halo is extended.

The orbit of these two rigid systems were computed assuming that:

- the force exerted by a rigid system (G1) on the other galaxy (G2) can be determined at the mass center of G2;

- the dynamical friction can be modeled by a viscous-like fluid drag force proportional to the local density (e.g., Combes et al. 1995). The force undergone by G1 is then assumed to be proportional to the density of $\mathrm{G} 2$ at the center of G1 $\rho_{2}\left(\boldsymbol{r}_{1}\right)$, and to $\boldsymbol{v}_{2}-\boldsymbol{v}_{1}$, where $\boldsymbol{v}_{i}$ is the velocity of $\mathrm{G}_{i}$. The force is then: $\boldsymbol{F}=k \rho_{2}\left(\boldsymbol{r}_{1}\right)\left(\boldsymbol{v}_{2}-\boldsymbol{v}_{1}\right)$. The proportionality factor $k$ was chosen for the dynamical friction time-scale at distance $50 \mathrm{kpc}$ to be $150 \mathrm{Myr}$, which is consistent with the results of previous $N$-body simulations. This description of the dynamical friction may seem very approximated, but $N$-body codes with a limited number of particles, and an arbitrary distribution of matter, also provide only an approximated friction.

We restricted our analysis to coplanar encounters, so that the gas particles evolve in a plane. In addition to the forces exerted on the two rigid galactic potential on gas particles in the frame of the three-body method, we used:

- a bidimensional FFT code to account for the gas self-gravity;

- a sticky-particles algorithm to model the dissipative dynamics of the ISM.
Both codes are described in Bournaud \& Combes (2002). We simulated galactic encounters with the same mass parameters for dark matter and the same initial gas distribution as in the full $N$-body simulations described in Sect. 2.

Figure 5 presents an example of a sequence of merging equal mass galaxies. Even in these simpler simulations, massive gaseous TDG progenitors of more than $10^{9} M_{\odot}$ are formed. Note that, in this run, the condensations are not necessarily located at the very tip of the tidal tails. At time $t=450 \mathrm{Myr}$, the condensation at $y>0$ is at about $2 / 3$ of the tail extremity. This is also observed in real interacting systems, such as the prototype merger NGC 7252 (Hibbard et al. 1994). Its HI tidal tail extends to the West further than its TDG candidate, located at the tip of the optical tail.

In Fig. 6, we show the effect of switching off the selfgravity and the gas dissipation, and hence the ability of gaseous tidal condensations to collapse. Large accumulations of gas are still present although they expectedly appear less compact and will probably not be able to survive for a long time in the numerical model. This clearly shows that the formation of massive TDGs is fundamentally a kinematical process and not the result of local gravitational instabilities that would later-on grow through accretion of surrounding material. The TDG progenitors are formed early on and already contain all the mass of the future TDG. Tidal forces within the potential well of extended dark matter haloes account for the large accumulations of tidally expelled gas. Only then, dissipation and self-gravity take over and trigger the cloud concentration and collapse.

One should note that, because of the kinematical origin of the proto-TDGs, our results are not sensitive to the fine-tuning of the gaseous dissipation rate which is, in numerical simulations, often critical to structure the tidal tails.

\subsection{The role of the DM halo extent}

\subsubsection{Response of a cold annulus to tidal forces}

In order to precisely understand the role of the DM haloes around the perturbing/perturbed galaxy, we analyzed how an annulus of cold material reacts to tidal forces. The simulations were carried out with the same code and parameters as in Sect. 3.1. Self-gravity has been disabled as we were only interested in studying the kinematical aspects of the TDG formation.

We first considered an annulus made of gas with zero velocity dispersion located at a constant radius $R_{0}$ in the parent galaxy. It is perturbed by the tidal forces exerted by the companion galaxy. We examined how particles that were initially uniformly distributed along the annulus react to the perturbation $380 \mathrm{Myr}$ after the beginning of the simulation, i.e. once the TDG progenitor is already visible, but before its collapse (see Fig. 5). As shown in Fig. 7, the annulus is transformed into a bent curve delineating the typical shape of a tidal tail. To make the analysis easier, we subtracted the effect of the differential rotation in the parent galaxy. In that new frame, tidal tails appear as linear structures, i.e. they have a mean constant azimuth, instead of being curved. In Fig. 8, we show the resulting plots in case of resp. extended and truncated DM haloes 

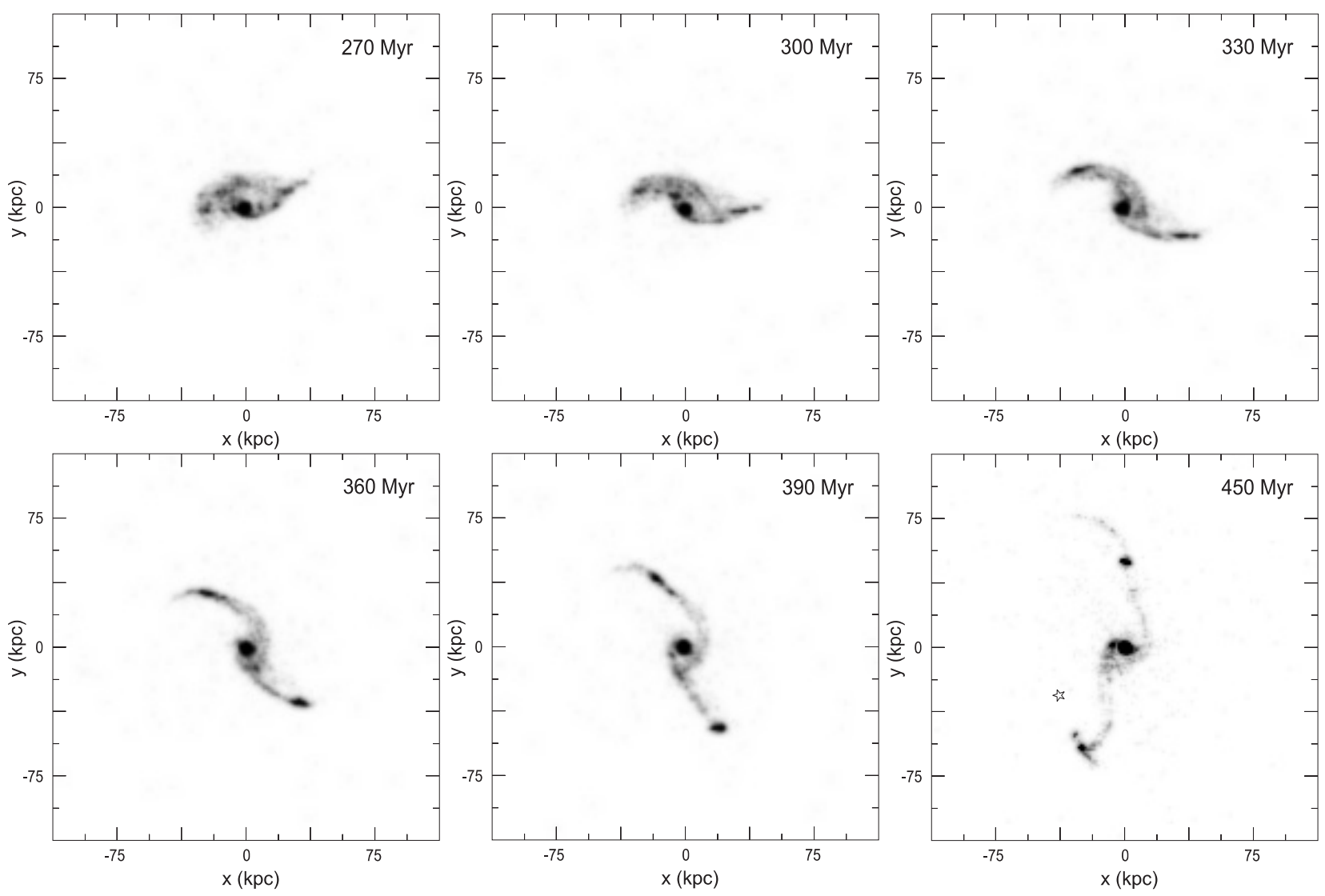

Fig. 5. Formation of the TDG progenitors in the simulations carried out with the restricted $N$-body + rigid haloes code. The evolution of the gas distribution in one of the merging galaxies is presented. The position of the the second galaxy is shown by a star at $t=450 \mathrm{Myr}$. At earlier times, it is outside the field of view. Self-gravity and energy dissipation in the ISM are included in these simulations. The parameters of the simulation were: mass ratio $1: 1,8 \%$ of gas, impact parameter $150 \mathrm{kpc}$, initial relative velocity $125 \mathrm{~km} \mathrm{~s}^{-1}$.
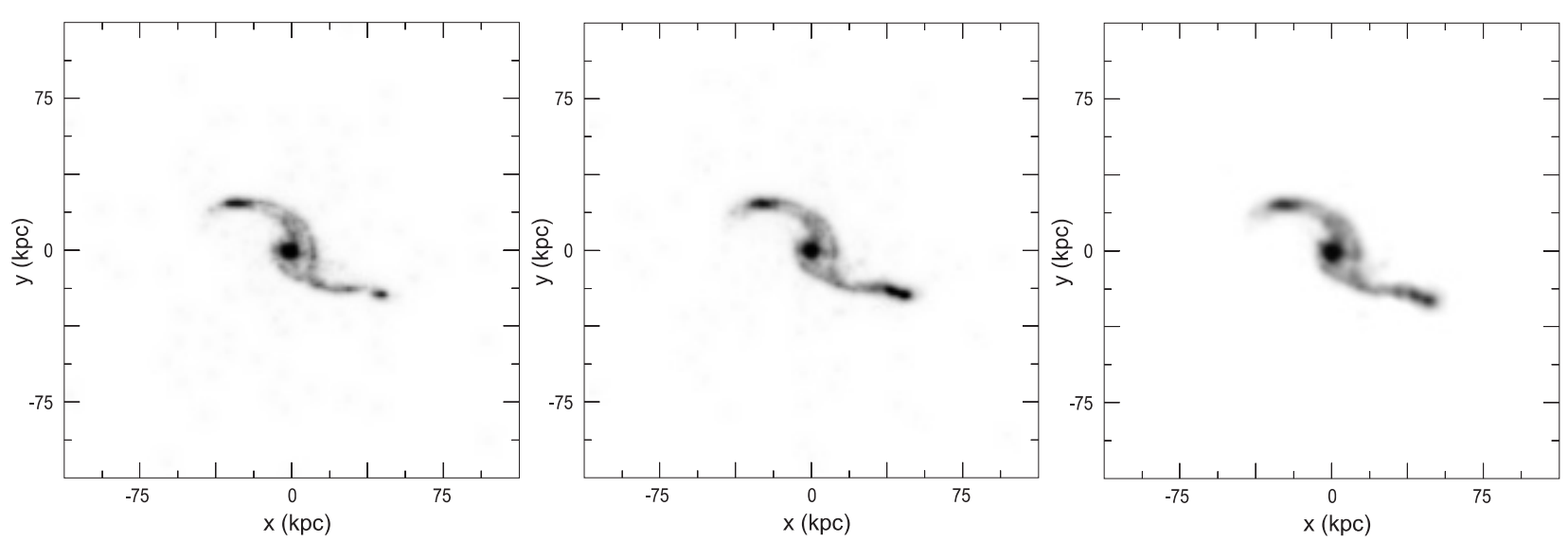

Fig. 6. Left: TDG gaseous progenitors at $t=345 \mathrm{Myr}$ for the collision shown in Fig. 5. Middle: same simulation suppressing the self-gravity in the gaseous component; right: same simulation suppressing self-gravity and energy dissipation.

around the parent and perturbing galaxies. In both cases, the tail gets denser at its extremity, i.e. the particles look closer to each other in the 1D space examined here. The amplitude of the overdensity, however, depends on the size of the DM haloes and is maximum when the haloes of both the parent galaxy and of the perturber are extended. The compression corresponds to the decrease of the radial distance $(\mathrm{d} r)$ and more significantly to the tightening of the azimuthal separation $(\mathrm{d} \theta)$ between the particles. In our simulations, $\mathrm{d} \theta$ is three times smaller when the haloes are extended.

\subsubsection{Amplitude of the radial excursions}

We then considered the more realistic situation of a set of concentric annuli with initial radii $R_{0}$ perturbed by tidal forces. We examined how the amplitude of the radial excursion, i.e. the 


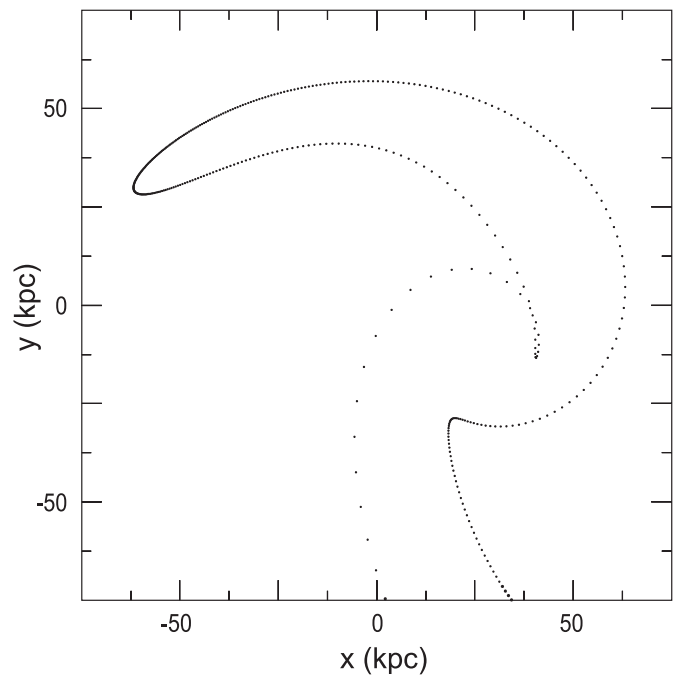

Fig. 7. Response of an annulus of uniformly distributed, kinematically cold, gas clouds to a tidal perturbation.

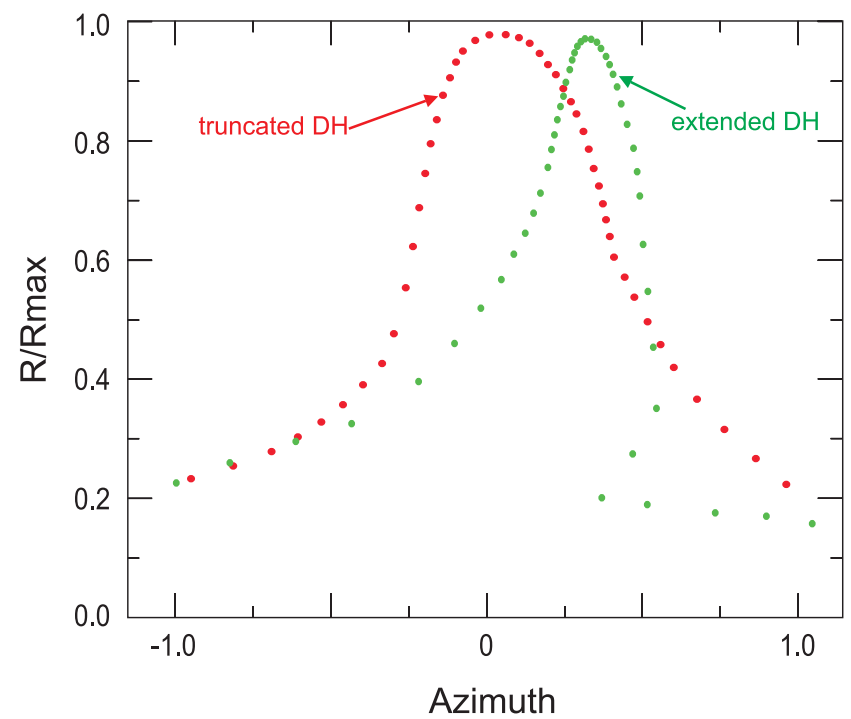

Fig. 8. Response of an annulus of uniformly distributed gas clouds to a tidal perturbation with DM haloes as extended as ten stellar radii (green solid line) and with DM haloes truncated at three stellar radii (red dashed line). The $x$-axis corresponds to the azimuth after correction of the differential rotation, so that the tidal tail has a fixed azimuth equal to zero. The radii $R$ have been scaled to the maximum extent of the tidal tail, $R \max$.

difference $\Delta R$ between the maximum radial extent (reached at the azimuth of the tidal tail) and the initial radius (see Fig. 9), varies with $R_{0}$. This will tell whether the $1 \mathrm{D}$ overdensities observed for each single annulus overlap and hence form a $2 \mathrm{D}$ condensation or whether they are uniformly distributed along the tail, canceling out any density contrast. Figure 9 illustrates the two situations. In Fig. 10 we show the evolution of $\Delta R$ as a function of $R_{0}$ for the truncated and extended haloes. In the first case, $\Delta R$ is a linear function of $R_{0}$, whatever $R_{0}$. Thus, the separation between two annuli in the tail is amplified and the overdensities are stretched along the tails. On the other hand, for extended haloes, $\Delta R$ is nearly constant beyond a

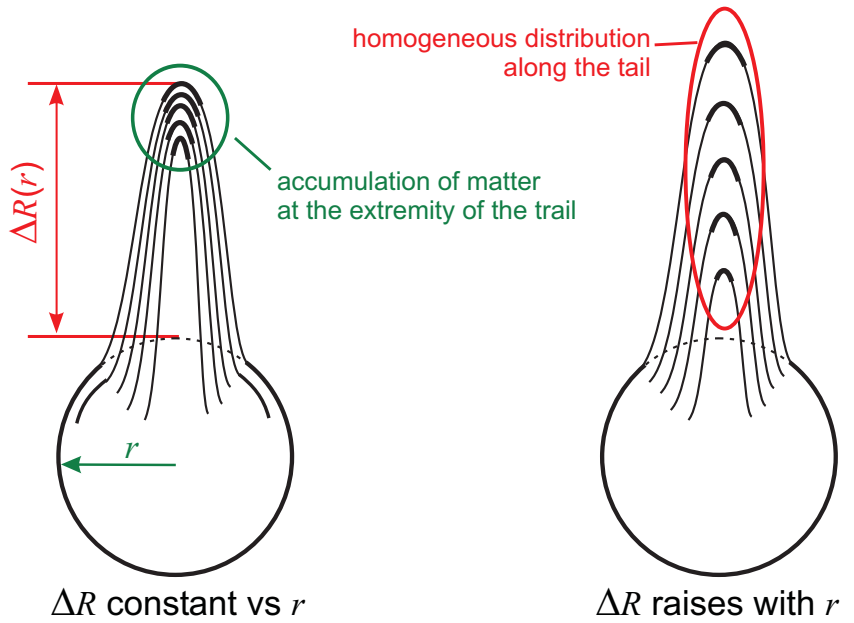

Fig. 9. Schematic view of the effects of tidal perturbations to a series of concentric annuli of radius $\mathrm{R}$ for extended (left) and truncated (right) dark matter haloes. The annuli were initially regularly spaced out.

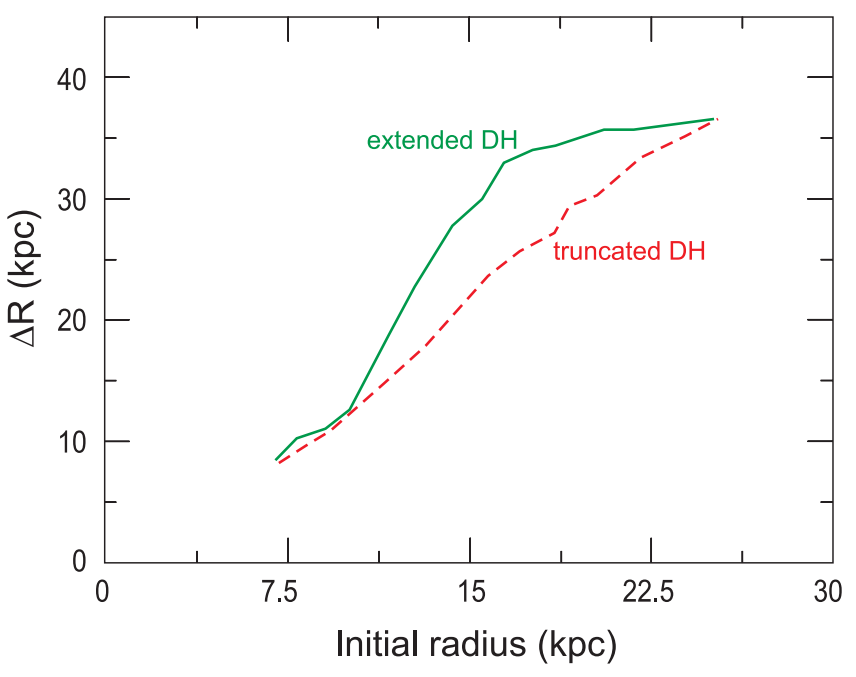

Fig. 10. Amplitude of the radial excursions as a function of the initial radius with DM haloes as extended as ten stellar radii (green solid line) and with DM haloes truncated at three stellar radii (red dashed line).

certain initial radius $(15 \mathrm{kpc}$ in the simulation shown in Fig. 10). Thus the distance between the annuli in the external regions of the parent galaxy is conserved and the overdensities are not diluted. Below this radius, the slope of the curve $\Delta R\left(R_{0}\right)$ is higher than in the truncated case, and hence, the matter is even more stretched along the tail. Both effects contribute to enhance the density contrast at the tip of the tail, and account for the accumulation of large quantities of matter there. In our simulations, the stretching along the radial direction, $\mathrm{d} \Delta R(R o) / \mathrm{d} R o$, is reduced by a factor of at least 7 (i.e., the compression enhanced by this value) for the 5 external kpc of the initial disk when the haloes are extended.

In summary, extended dark haloes cause, for a given annulus, a compression of matter at the extremity of the future tidal tail (smaller $\mathrm{d} \theta$ ), and a smaller separation between several concentric annuli (smaller $\mathrm{d} \Delta R(R o) / \mathrm{d} R o$ ). Thus, matter appears 
more concentrated both in the azimuthal and radial directions than with truncated dark haloes.

The physical explanation for the kinematical formation of a TDG progenitor lies in the shape of the tidal forces exerted on matter depending on its location with respect to the DM haloes. A mathematical description of the tidal field and its effect on test particles is given below.

\subsection{The shape of the tidal field}

The potential field outside of a truncated halo is a Keplerian potential, as arising from a point-like mass, while the potential field inside of an extended halo can be accounted for, with a good approximation, by an isothermal sphere potential. One reason why a Keplerian potential and an isothermal sphere potential lead to so qualitatively different outcomes is likely linked to the properties of the tidal field of a spherical potential $\phi(r)$ (Dekel et al. 2003).

Assuming the potential center lies at $X=-r_{0}, Y=0$ and $Z=0$ in a Cartesian $(X, Y, Z)$ frame, we can evaluate the corresponding tidal field defined as the differential acceleration between a test particle located at an arbitrary position $X, Y, Z$ and a test particle located at the frame origin. To lowest order in $X / r_{0}$, $Y / r_{0}$ and $Z / r_{0}$, the components of the tidal field read:

$\gamma_{X}=-\left(\partial_{r^{2}}^{2} \phi\right) X$

$\gamma_{Y}=-\left(1 / r_{0}\right)\left(\partial_{r} \phi\right) Y$

$\gamma_{Z}=-\left(1 / r_{0}\right)\left(\partial_{r} \phi\right) Z$.

From these equalities one can see directly that for a Keplerian potential $\left(\phi \propto r^{-1}\right)$ one has $\gamma_{Y} / Y=\gamma_{Z} / Z=-(1 / 2) \gamma_{X} / X$, while for an isothermal sphere potential (for which $\phi \propto \log r$ ), one has: $\gamma_{Y} / Y=\gamma_{Z} / Z=-\gamma_{X} / X$. In particular, if one is interested in motions restricted to the $(X, Y)$ plane, then the tidal field is divergence free for an isothermal sphere potential (i.e. inside a dark matter halo), while this divergence is positive for a Keplerian potential (i.e. outside of a dark matter halo). In other words, the tidal field of a Keplerian potential tends not only to stretch fluid elements, but also to dilute them, whereas the tidal field of an isothermal sphere tends to stretch fluid elements while conserving their surface density.

In order to further investigate this role of the tidal field shape, we have performed simple calculations of a cold bidimensional disk of test particles orbiting within its own extended halo represented by an isothermal sphere potential (i.e. with a flat rotation curve). It is perturbed by an external, time varying, tidal field, as would arise from a perturbing galaxy.

The tidal field is expressed by:

$\gamma_{X}=\lambda_{1} f(r) X$

$\gamma_{Y}=\lambda_{2} f(r) Y$,

where the frame $(X, Y)$ is centered on the disk and has its $X$-axis oriented toward the fictitious by-passing perturber located at distance $r(t)$ from the center of the disk. The two numerical constants $\lambda_{1}$ and $\lambda_{2}$ describe the shape of the tidal field and therefore correspond to its local properties, while the function $f(r)$ describes how the tidal field intensity decays with the

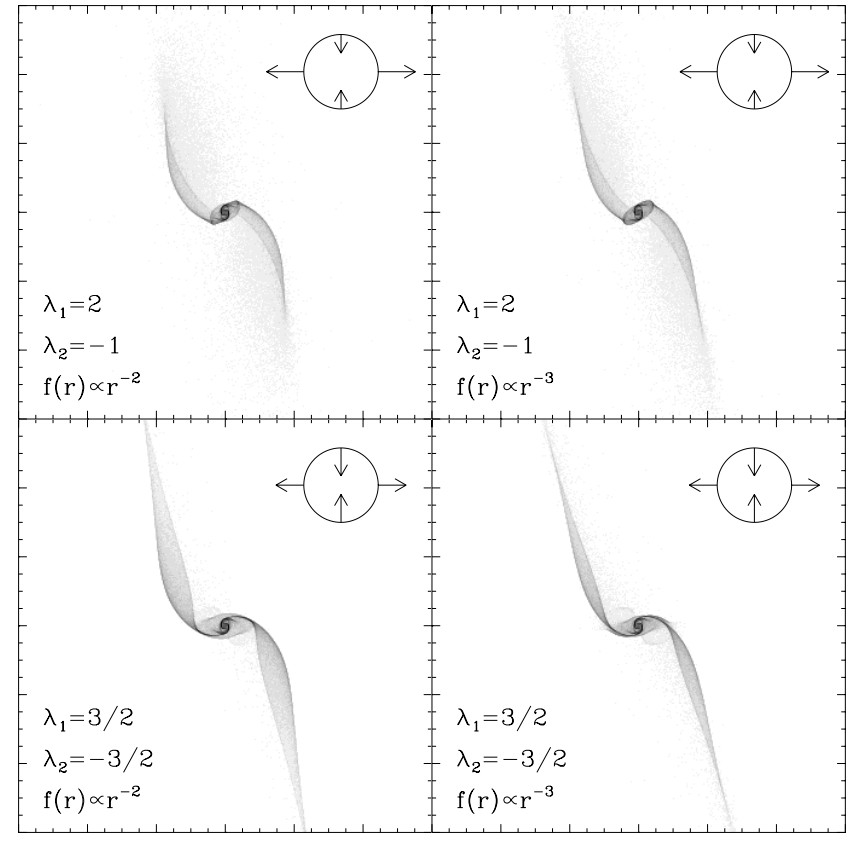

Fig. 11. Plots in the left column correspond to the decay of an extended halo potential $\left(f(r) \propto r^{-2}\right)$ while those of the right column correspond to a Keplerian decay $\left(f(r) \propto r^{-3}\right)$ of the tidal field. Similarly, the runs of the top row correspond to a local shape of the tidal field that is Keplerian $\left(\lambda_{1}=-2 \lambda_{2}\right)$, while the runs of the bottom row correspond to a local shape of the tidal field corresponding to an isothermal sphere potential $\left(\lambda_{1}=-\lambda_{2}\right)$. By comparing these four plots, one can conclude that it is the relative value of $\lambda_{1}$ and $\lambda_{2}$ that primarily determines the arms morphology, i.e. the local shape of the tidal field. The grey levels correspond to the same values on the four plots, and scale with the square root of the surface density. Notice in particular the presence of a diffuse scattered population of test particles in the cases where the tidal potential has a Keplerian shape (top row). The symbols in each panel represent the shape of the tidal field as in Dekel et al. (2003).

distance to the perturber $\left(f(r) \propto r^{-2}\right.$ for an isothermal sphere potential, while $f(r) \propto r^{-3}$ for a Keplerian potential), and is therefore a global property of the tidal field. The $\lambda_{1}$ and $\lambda_{2}$ constants are actually imposed by the $f(r)$ function, through the Eqs. (2). It is however instructive to artificially set $\lambda$ and $f(r)$ separately in order to disentangle the different effects at work in exciting the tidal arms.

Namely, we have run several calculations for which we either set $\lambda_{1}=2, \lambda_{2}=-1$ (which corresponds to a Keplerian tidal field shape), or $\lambda_{1}=3 / 2, \lambda_{2}=-3 / 2$ (which corresponds to an isothermal sphere tide shape), everything else being fixed (in particular the $f(r)$ function). We find with this toy model that the tidal arms corresponding to this second choice are systematically more pronounced and longer than the tidal arms excited by a Keplerian tidal field. In a similar manner, we have performed calculations in which we either have $f(r) \propto r^{-2}$ or $f(r) \propto r^{-3}$, everything else being fixed. Although the tidal arms position angle might vary slightly between two such calculations, they display similar lengths and more significantly similar surface densities, which enables us to conclude that it is primarily the shape of the tidal field (i.e. the relative value of $\lambda_{1}$ and $\lambda_{2}$ ) that is important in shaping and structuring the tidal arms. Figure 11 illustrates this conclusion. It represents at 
the same date the surface density of a disk perturbed by a tidal field of the type given by Eq. (3), with different prescriptions for $\left(\lambda_{1}, \lambda_{2}\right)$ and $f(r)$.

Wallin (1990) made a similar study of the structure of tidal tails, based on restricted 3-body simulations. He noted a density increase in the tidal tails caused by their twisting and the formation of orbital caustics on their outside edge. Such caustics which correspond to the crossing of particle trajectories are also visible in Fig. 11. Like a density wave, the twist creates a local overdensity which propagates outwards. Meanwhile the amplitude of the density enhancement decreases. Wallin (1990) explored a wide variety of initial conditions and orbital parameters, but did not explicitly examined how his results were affected by the shape of the tidal field, as influenced by the size of the DM haloes. He speculated that, when the mass distribution is extended, the orbital speed of the companion decreases, and the propagation of the twist is delayed and slowed down.

Our simple calculations however strongly suggest that it is the special shape of an extended halo tidal field $\left(\lambda_{1}=-\lambda_{2}\right)$ that is crucial to enable the formation of TDGs.

These results agree with what we previously found: when the tidal interaction occurs outside truncated dark haloes, the divergent tidal field inhibits the formation of massive accumulations of matter. When the interaction occurs inside extended dark haloes, the tidal field can efficiently carry away from the disk a large fraction of the gas, while maintaining its surface density to a high value. This results in long, contrasted tidal tails, at the end of which further processes can lead to the formation of TDG-like objects.

\section{Discussion}

\subsection{Top-down versus bottom-up scenario}

The formation mechanism of classical galaxies has long been a puzzle. Whether they form from the merging of smaller substructures, i.e. dwarf galaxies, - the bottom-up scenario -, or from the collapse of larger structures - the top-down scenario-, has been actively debated. The widely supported Cold Dark Matter model now promotes the hierarchical scenario where small objects form first, although a monolithic collapse is still regularly claimed, especially to account for the formation of the old ellipticals in clusters.

Even if Tidal Dwarf Galaxies have a very different origin, the discussion on their formation mechanism may, to a large extent, be brought up in similar terms. The early numerical simulations by Barnes \& Hernquist (1992) and Elmegreen et al. (1993) showed the formation of bound structures all along the tidal tails, with typical masses of $10^{7}-10^{8} M_{\odot}$. One may have wondered whether they were the progenitors of the more massive (and gaseous) TDGs observed in some interacting systems. Indeed they could in principle accrete surrounding tidal material, in particular gas clouds. However, the uniform distribution of the bound clumps along the tails argue against this scenario, at least for the massive TDGs found near the tip of the tails.

Our simulations indicate that, whenever the dark matter haloes around the parent galaxies are sufficiently extended (their radii are at least 10 times that of the optical radius, or $150 \mathrm{kpc}$ for a typical spiral galaxy), disk material coming from outside a given radius may pile up at or near the end of the tidal tails, instead of being stretched along them. Thus, a kinematical process, rather than a local growing instability, seems to be at the very origin of the progenitors of massive TDGs. Because the gas is more extended than the stars in the parent's disk, the proto-TDGs are initially mainly made of gas. Later on, the clouds collapse under the effect of gravity and start forming stars. At the end of our $N$-body simulations, the bulk of the stellar population is made of stars born in situ in the tail. In a few runs, the progenitor broke into two fragments with individual masses still above or around $10^{9} M_{\odot}$. Therefore, the formation of TDGs is more consistent with a top-down scenario.

\subsection{Redefining Tidal Dwarf Galaxies}

Massive TDGs are not the only objects formed out of tidal debris. We enumerated in the introduction many other by-products of galaxy collisions, such as Super Star Clusters, Young Globular Clusters, Giant HII Complexes, etc. Many of them are actually called TDG candidates in the literature. Overall, the definition of a TDG remains elusive. A basic consensual characteristic of any object defined as a TDG is that it should be a gravitationally bound system (Duc et al. 2000), although such a property is, observationally, difficult to assess (Hibbard et al. 2001). High resolution kinematical studies may help to find evidences for a kinematical independence of tidal objects (Duc \& Mirabel 1998; Mendes de Oliveira et al. 2001; Weilbacher et al. 2003; Bournaud et al. 2004). Now, not all self-gravitating structures in tidal tails have the required initial mass to form a genuine galaxy. Taking into account evaporation and fragmentation processes plus tidal disruption, a total mass as high as $10^{9} M_{\odot}$ may be necessary. This is the typical mass of the giant HI accumulations observed near the tip of several long tidal tails. Less massive condensations may evolve, if they survive, into objects more similar to globular clusters.

However, if all tidal objects were formed by the same physical mechanism, fixing a mass threshold for defining a TDG would appear arbitrary. Beside, a weakness of such a definition is that we do not a priori know how a proto-TDG evolves. Numerical simulations have just now the required resolution to study the precise fate of tidal debris (see next section), while, observationally, no "old", independent TDG, detached from the parent galaxies, has yet been unambiguously identified (Duc et al. 2004). Their final mass and morphology a dwarf spheroidal, as suggested by Kroupa (1998)? - is then questionable.

The results of our simulations and their modeling provide a stronger theoretical support for distinguishing the massive gas accumulations near the tip of tidal tails and the other less massive condensations along them. Indeed, as stated in Sect. 4.1, they seem to have formed by two different mechanisms. We therefore wish to define as proto-TDGs only the objects of the first category, i.e. those made of tidal material that was able to accumulate in the tail and later collapse, according to a topdown scenario. Indeed, this process is the only one able to form condensations with masses greater than $10^{9} M_{\odot}$, that are 
enough distant $(>50 \mathrm{kpc}$ ) from their parent galaxies to avoid a rapid fall back (Hibbard \& Mihos 1995) or a tidal disruption. In contrast, the clumps along the tails have basically a gravitational origin and formed from instabilities in either the stellar (Barnes \& Hernquist 1992) or gaseous (Elmegreen et al. 1993) components.

Adopting this definition, one should admit that only specific, restrictive, conditions are required to form a TDG, in particular orbital parameters of the collision that favor the development of long tidal tails, and the presence of extended dark matter haloes around the parent galaxies. Both criteria may actually be simultaneously fulfilled. Indeed Springel \& White (1999) noted that less extensive haloes make weaker tails. Another consequence is that only one proto-TDG per tail may be formed. However, it may later fragment (as seen in Fig. 4) and generate a few extra TDGs. Determining the production rate of TDGs in merging systems is beyond the scope of this paper. It would require to systematically explore the parameter space of the collisions (varying the orbital parameters, structural properties of the parent galaxies, their mass ratios, etc .). We are currently engaged in such a long term program.

Do these conditions exclude that TDGs and the less massive SSCs form simultaneously? This is not necessarily the case. In the simulations shown in Fig. 4 (especially run D), a few faint clumps can be seen along one of the the tails. They look like the bound objects produced in the simulations by Elmegreen et al. (1993), although they are much less numerous in our simulations. Indeed, because a TDG already collects a large fraction of the tidal material (about 75\%), there remains much less of it for the SSCs progenitors. This would be consistent with the observation by Knierman et al. (2003) that massive and compact condensations do not cohabit within a single tidal tail.

\subsection{Evolution of the proto-TDGs}

In the preliminary low resolution simulations presented in BDM03, we noted that the TDGs survive for more than 1.5 Gyr and end up orbiting around the merger remnant, like any satellite galaxy. This result is in agreement with the study of Hibbard \& Mihos (1995) who, studying the return of tidal material in the prototype merger NGC 7252, concluded that particles initially sent away at more than $100 \mathrm{kpc}$ would not fall back within a Hubble time. Our new full $N$-body simulations have a higher resolution, include feedback but were ended earlier. Still, we found that $0.5 \mathrm{Gyr}$ after the formation of their progenitors, the two TDGs already appear isolated, orbiting at radial distances of about $100 \mathrm{kpc}$ (see Fig. 3). Meanwhile, they have already used up several tens of percent of their gas reservoir and transformed them into young stars. This stellar population born in situ dominates the older component tidally stripped from the parent galaxies. Which confidence, however, can we give to these results on the evolution of TDGs?

The resolution of our full $N$-body simulations is as a matter of fact still insufficient to firmly predict the survival time of the TDGs. Each TDG progenitor is modeled by typically $10^{5}$ gas particles and $10^{4}$ star particles. This is just enough to model the destructive feedback effect (due to its internal starburst). A refined grid around the TDG would be required to properly take into account the tidal shear in the young galaxy (induced by the parent galaxy) and, possibly, the fragmentation of its progenitor. Increasing the resolution of our simulations towards forming TDGs, we should hopefully soon infer the real impact of these additional processes on their evolution and life expectancy.

\section{Conclusions}

In this paper, we presented a set of numerical simulations of various types aimed at studying the structure of tidal tails and their ability to concentrate prominent condensations likely to become Tidal Dwarf Galaxies. Our immediate objective was to check the results obtained by Bournaud et al. (2003) with a simple numerical model: massive TDG progenitors may form provided that the dark matter haloes around their parent galaxies are very extended, at least ten times more than the stellar disk. Carrying out further tests, we reached the following conclusions:

- Gas accumulations as massive as $10^{9} M_{\odot}$ are still formed near the end of tidal tails in $N$-body codes that include a large number of test particles, self-gravity, dissipation and feedback. Our initial results were therefore robust.

- Switching off the self-gravity and dissipation in simpler $N$-body simulations with rigid haloes does not inhibit the gathering of tidal debris. This implies that the formation of massive tidal accumulations is fundamentally due to a kinematical process: tidal forces make a significant fraction of the gas coming from the outer parent's disk to pile up near the extremity of the tail. Once enough material has accumulated, self-gravity may lead to the local collapse of the gas clouds.

- Using toy models, we clarified the role of the dark matter haloes around the parent galaxies. We first analyzed how a 1D annulus of cold gas that was originally uniformly distributed responds to a tidal perturbation. We observed an overdensity at the tip of the tidal tail that is much more pronounced when the dark matter haloes around both the perturbed galaxy and the perturber are extended. We further determined the amplitude of the radial excursions exerted on a set of concentric annuli. For truncated DM haloes, the tidal material, and hence the individual 1D overdensities, are stretched all along the tail. On the other hand, when the DM haloes are extended, this dilution only occurs below an initial radius. The density contrast at the tip of the tail is then enhanced.

- Test particles expelled outside a truncated halo are subject to a Keplerian potential while the particles that will remain inside the extended halo feel an isothermal sphere potential. The differences in the resulting tidal fields account for the different efficiencies with which large quantities of gas may be carried away. We found that the local shape of the tidal field plays the most important role.

- The objects born in the prominent gas accumulations were formed according to a top-down scenario, contrary 
to the less massive Giant HII complexes and Super Star (Globular) Clusters that also formed along tidal tails from growing local instabilities. Having different physical origins, these different classes of collisional by-products have also different fates.

- We wish to define as Tidal Dwarf Galaxies (TDGs) only those objects having a progenitor exceeding $10^{9} M_{\odot}$, formed out of collapsing gaseous material that accumulated far enough from the parent galaxies, usually (but not necessarily) near the tip of the tidal tails. Indeed, they are the most likely to survive and become genuine satellite galaxies. Such TDGs can only form under specific conditions, in particular collisions that favor the development of long tidal tails within extended dark matter haloes. They were observed so far in a limited number of interacting systems, but a systematic census of such objects is still to be done.

More extensive simulations of galaxy collisions are required to assess the cosmological importance of TDGs. The parameter space of the orbits and initial physical conditions in the parent galaxies should be more thoroughly explored to estimate the production rate of the proto-TDGs. Using a more refine grid would help to study the evolution of the TDGs and determine their life expectancy.

Acknowledgements. The numerical simulations were carried out on the NEC-SX6 vectorial computer of the CCRT of CEA. We are most grateful to Romain Teyssier for stimulating discussions on these simulations and to Françoise Combes for her advice on this project and her comments on the manuscript.

\section{References}

Barnes, J. E. 1992, ApJ, 393, 484

Barnes, J. E., \& Hernquist, L. 1992, Nature, 360, 715

Barnes, J. E., \& Hernquist, L. 1996, ApJ, 471, 115

Bekki, K., Forbes, D. A., Beasley, M. A., \& Couch, W. J. 2002, MNRAS, 335, 1176

Bournaud, F., \& Combes, F. 2002, A\&A, 392, 83

Bournaud, F., \& Combes, F. 2003, A\&A, 401, 817

Bournaud, F., Duc, P.-A., \& Masset, F. 2003, A\&A, 411, L469

Bournaud, F., Duc, P., Amram, P., et al. 2004, A\&A, 425, 813

Braine, J., Duc, P.-A., Lisenfeld, U., et al. 2001, A\&A, 378, 51

Combes, F., Boisse, P., Mazure, A., et al. 1995, Galaxies and Cosmology (Berlin, Heidelberg, New York: Springer-Verlag), also Astronomy and Astrophysics Library

Cortese, L., Gavazzi, G., Boselli, A., \& Iglesias-Paramo, J. 2004, A\&A, 416, 119

de Grijs, R., Lee, J. T., Clemencia Mora Herrera, M., et al. 2003, New Astron., 8, 155

Dekel, A., Devor, J., \& Hetzroni, G. 2003, MNRAS, 341, 326

Dubinski, J., Mihos, J. C., \& Hernquist, L. 1996, ApJ, 462, 576

Dubinski, J., Mihos, J. C., \& Hernquist, L. 1999, ApJ, 526, 607

Duc, P.-A., \& Mirabel, I. F. 1998, A\&A, 333, 813

Duc, P.-A., \& Mirabel, I. F. 1999, in IAUS 186: Galaxy Interactions at Low and High Redshift, ed. J. E. Barnes, \& D. B. Sanders (Dordrecht: Kluwer), 61
Duc, P.-A., Brinks, E., Wink, J. E., \& Mirabel, I. F. 1997, A\&A, 326, 537

Duc, P.-A., Brinks, E., Springel, V., et al. 2000, AJ, 120, 1238

Duc, P.-A., Bournaud, F., \& Masset, F. 2004, in IAU Symp., 217, Recycling intergalactic and interstellar matter, ed. P. A. Duc, J. Braine, \& E. Brinks, ASP, 550 [arXiv: astro-ph/0402252]

Elmegreen, B. G., Kaufman, M., \& Thomasson, M. 1993, ApJ, 412, 90

English, J., Norris, R. P., Freeman, K. C., \& Booth, R. S. 2003, AJ, 125,1134

Gallagher, S. C., Charlton, J. C., Hunsberger, S. D., et al. 2001, AJ, 122,163

Gerhard, O., Arnaboldi, M., Freeman, K. C., \& Okamura, S. 2002, ApJ, 580, L121

Gilbert, A. M., Graham, J. R., McLean, I. S., et al. 2000, ApJ, 533, L57

Hibbard, J. E., \& Mihos, J. C. 1995, AJ, 110, 140

Hibbard, J. E., \& van Gorkom, J. H. 1996, AJ, 111, 655

Hibbard, J., \& Barnes, J. E. 2004, in IAU Symp., 217, Recycling intergalactic and interstellar matter, ed. P. A. Duc, J. Braine, \& E. Brinks, ASP, 510

Hibbard, J. E., Guhathakurta, P., van Gorkom, J. H., \& Schweizer, F. 1994, AJ, 107, 67

Hibbard, J. E., van der Hulst, J. M., Barnes, J. E., \& Rich, R. M. 2001, AJ, 122, 2969

Holtzman, J. A., Faber, S. M., Shaya, E. J., et al. 1992, AJ, 103, 691

Jungwiert, B., Combes, F., \& Palouš, J. 2001, A\&A, 376, 85

Knierman, K. A., Gallagher, S. C., Charlton, J. C., et al. 2003, AJ, 126, 1227

Kroupa, P. 1998, MNRAS, 300, 200

López-Sánchez, Á. R., Esteban, C., \& Rodríguez, M. 2004, ApJS, 153 , 243

Mendes de Oliveira, C., Plana, H., Amram, P., et al. 2001, AJ, 121, 2524

Mendes de Oliveira, C., Cypriano, E. S., Sodré, L., \& Balkowski, C. 2004, ApJ, 605, L17

Mihos, C. 2004, in IAU Symp., 217, Recycling intergalactic and interstellar matter, ed. P. A. Duc, J. Braine, \& E. Brinks, ASP, 390 [arXiv: astro-ph/0401557]

Mihos, J. C., Dubinski, J., \& Hernquist, L. 1998, ApJ, 494, 183

Mirabel, I. F., Dottori, H., \& Lutz, D. 1992, A\&A, 256, L19

Roberts, M. S., \& Haynes, M. P. 1994, ARA\&A, 32, 115

Ryan-Weber, E. V., Meurer, G. R., Freeman, K. C., et al. 2004, AJ, 127,1431

Saviane, I., Hibbard, J. E., \& Rich, R. M. 2004, AJ, 127, 660

Schmidt, M. 1959, ApJ, 129, 243

Schweizer, F., Miller, B. W., Whitmore, B. C., \& Fall, S. M. 1996, AJ, 112,1839

Springel, V., \& White, S. D. M. 1999, MNRAS, 307, 162

Toomre, A., \& Toomre, J. 1972, ApJ, 178, 623

Tran, H. D., Sirianni, M., Ford, H. C., et al. 2003, ApJ, 585, 750

Wallin, J. F. 1990, AJ, 100, 1477

Weilbacher, P. M., Duc, P.-A., \& Fritze-v. Alvensleben, U. 2003, A\&A, 397, 545

Whitmore, B. C., Zhang, Q., Leitherer, C., et al. 1999, AJ, 118, 1551

Zepf, S. E., Ashman, K. M., English, J., et al. 1999, AJ, 118, 752 